\title{
HSP90 interacts with HMGCR and promotes the progression of hepatocellular carcinoma
}

\author{
LI DONG $^{1}$, LIYING XUE ${ }^{1}$, CUIYING ZHANG ${ }^{1}$, HUI LI $^{1}$, ZHIHUI CAI $^{1}$ and RUIFANG GUO ${ }^{2}$ \\ ${ }^{1}$ Department of Medical Oncology; ${ }^{2}$ Clinical Nutrition Center, Inner Mongolia People's Hospital, \\ Hohhot, Inner Mongolia 010017, P.R. China
}

Received October 30, 2016; Accepted April 13, 2018

DOI: $10.3892 / \mathrm{mmr} .2018 .9667$

\begin{abstract}
Heat shock protein 90 (HSP90) has been reported to promote the growth and inhibit apoptosis of hepatocellular carcinoma (HCC) cells. However, the underlying mechanisms are not fully understood. Immunostaining of the tissue array demonstrated that HSP90 was upregulated in HCC clinical samples and was associated with clinical features. HSP90 interacted with 3-hydroxy-3-methylglutaryl-CoA reductase (HMGCR), the rate-limiting enzyme of mevalonate pathway, in the immunoprecipitation assay and regulated its protein expression level by inhibiting protein degradation. In addition, lovastatin, an inhibitor of HMGCR, impaired the oncogenic functions of HSP90 in the cell growth, migration and colony formation assays. Taken together, this study demonstrated that HSP90 promoted the progression of HCC by positively regulating the mevalonate pathway and indicated that HSP90 may be a promising therapeutic target.
\end{abstract}

\section{Introduction}

Hepatocellular carcinoma (HCC) is one of the most common malignancies worldwide (1). Although great progress has been achieved in the diagnosis and treatment of HCC, the overall survival of HCC patients remains poor (2). Alteration of metabolism is a hallmark of cancer cells (3). Thus, targeting metabolic pathways may be a promising strategy for the treatment of HCC.

The mevalonate (MVA) pathway is responsible for cholesterolsynthesisinmammals (4).3-Hydroxy-3-methylglutarylCoA reductase (HMGCR) is a rate-limiting enzyme for the MVA pathway (5). Numerous studies have demonstrated the oncogenic roles of the MVA pathway in tumorigenesis $(6,7)$. It has been reported that enzymes involved in the MVA pathway

Correspondence to: Dr Ruifang Guo, Clinical Nutrition Center, Inner Mongolia People's Hospital, 20 Zhaowuda Road, Saihan, Hohhot, Inner Mongolia 010017, P.R. China

E-mail: guoruifang2016@163.com

Key words: heat shock protein 90,3-hydroxy-3-methylglutaryl-CoA reductase, lovastatin, cell growth and migration were upregulated in breast cancer and HMGCR promoted the transformation of benign breast epithelium (2). In gastric cancer cells, HMGCR has been reported to activate the hedgehog signaling pathway and promotes the growth and migration of cancer cells (8). In HCC, HMGCR has been demonstrated to regulate the activation of yes-associated protein and the chemoresistance of cancer cells (2). The protein expression level of HMGCR is tightly controlled at multiple levels. Sterol regulatory element-binding protein 2 is a key modulator of HMGCR via regulation of its transcription (9). On the other hand, ubiquitin fusion degradation (UFD) and autocrine motility factor receptor (GP78) promoted the degradation of the HMGCR protein (10). In addition, several microRNAs have been reported to target HMGCR (11). Statins, which are HMGCR inhibitors, have attracted attention for their role in lowing cholesterol and anti-cancer activity (2). Therefore, increased understanding of the regulation of HMGCR may improve the treatment of HCC.

Heat-shock protein 90 (HSP90) is a molecular chaperon and serves an important role in the protein folding, stability and degradation of proteins referred to as 'client proteins' (12). Numerous oncogenes (including epidermal growth factor receptor, human epidermal growth factor receptor and protein kinase B) are client proteins (13-15). Due to environmental stress (hypoxia, low $\mathrm{pH}$ and lack of nutrients), cancer cells are more dependent on the function of HSP90, which may explain the significant upregulation of HSP90 in cancer tissues $(16,17)$. Several studies have reported the function of HSP90 in HCC by regulating cell proliferation and apoptosis, and the inhibitor for HSP90, 17-DMAG, has shown anti-cancer activity $(18,19)$. However, the detail underlying mechanism of the function of HSP90 in HCC remain largely unknown. This study examined the expression pattern of HSP90 in HCC and investigated the underlying molecular mechanism.

\section{Materials and methods}

Cell culture. L02, 7404, huh-7, Hep3B, QGY and MHCC97 cell lines were obtained from American Type Culture Collection (Manassas, VA, USA). Cells were cultured in Dulbecco's Modified Eagle's medium (DMEM; Invitrogen; Thermo Fisher Scientific, Inc., Waltham, MA, USA) containing antibiotics $(100 \mathrm{U} / \mathrm{ml}$ penicillin and $0.1 \mathrm{mg} / \mathrm{ml}$ streptomycin; Sigma-Aldrich; Merck KGaA, Darmstadt, Germany) and 10\% 
fetal bovine serum (FBS; Sigma-Aldrich, Merck KGaA), and incubated at $37^{\circ} \mathrm{C}$ in an atmosphere containing $5 \% \mathrm{CO}_{2}$.

Clinical HCC samples and tissue array. Human clinical HCC samples were collected from patients that received surgery at Inner Mongolia People's Hospital (Hohhot, China). This study was approved by the ethics committee of Inner Mongolia People's Hospital, and written informed consent was obtained from patients. Tissues were stored at $-80^{\circ} \mathrm{C}$. The tissue microarray was constructed by the Shanghai Outdo Biotech Co., Ltd. (Shanghai, China), which included 297 patients (266 males and 31 females; aged 58-68 years) who received surgery at Inner Mongolia People's Hospital between 01/07/2009 and 06/04/2012. Patients did not receive chemotherapy or radiotherapy prior to operation.

Reverse transcription-quantitative polymerase chain reaction (RT-qPCR). Total RNA and miRNA was extracted from the cell lines using TRIzol (Thermo Fisher Scientific, Inc.). RT was performed using RNA to cDNA EcoDry Premix (Takara Biomedical Technology (Beijing) Co., Ltd., Beijing, China), according to the manufacturer's protocol. Subsequently, qPCR was performed in triplicate with a QuantiTect SYBR Green PCR kit (Qiagen GmbH, Hilden, Germany). The PCR protocol was as follows: $95^{\circ} \mathrm{C}$ for $5 \mathrm{~min}$, followed by 40 cycles of $95^{\circ} \mathrm{C}$ for $30 \mathrm{sec}, 60^{\circ} \mathrm{C}$ for $30 \mathrm{sec}$ and $68^{\circ} \mathrm{C}$ for $3 \mathrm{~min}$. Following a final hold step at $68^{\circ} \mathrm{C}$ for $10 \mathrm{~min}$, the samples were stored at $10^{\circ} \mathrm{C}$. The relative gene expression levels were calculated using the $2^{-\Delta \Delta \mathrm{Cq}}$ method (20). Primers for HSP90 were as follows: Forward, 5'-GTTAACTGGTACCAAGAAAA-3' and reverse, 5'-CGGATTTTGTCCAATGCATC-3'. Primers for GAPDH were as follows: Forward, 5'-GGAGCGAGATCCCTCCAA AAT-3' and reverse, 5'-GGCTGTTGTCATACTTCTCAT GG-3'.

Plasmid construction. The coding sequence of HMGCR was inserted into pcDNA3.1 (Addgene, Inc., Cambridge, MA, USA) containing myc tag by HindIII and EcoRV, the coding sequence of HSP90 was inserted into P23 (Addgene, Inc.) containing Flag tag and green fluorescent protein by $\mathrm{XbaI}$ and EcoRI, and the coding sequence of UFD was inserted into pCMV-HA (Addgene, Inc.) using PstI and BamHI. All restriction enzymes were obtained from Takara Biomedical Technology (Beijing) Co., Ltd. An empty vector was used as the control. The expression vectors were transfected into 7404 and Huh-7 cells using Lipofectamine ${ }^{\circledR} 2000$ reagent (Thermo Fisher Scientific, Inc.) for $8 \mathrm{~h}$ at $37^{\circ} \mathrm{C}$, following the manufacturer's protocol. A total of $3 \times 10^{5}$ cells were seeded in 6-well plates and cells were transfected with $1 \mu \mathrm{g} / \mu \mathrm{l}$ plasmids using Lipofectamine ${ }^{\circledR} 2000$. Cells were selected using fluorescence-activated cell sorting by detecting expression of green fluorescent protein (independently expressed).

Knocking down the expression of HMGCR in HCC cells. The lentivirus (multiplicity of infection=1) used to knock down the expression of HMGCR was purchased from Shanghai GeneChem Co., Ltd. (Shanghai, China). The sequences for HMGCR-short hairpin (sh)RNA were as follows: Forward, 5'-AAGTCATAGTGGGGACAGTGA-3'; reverse, 5'-AAA CTCTGATGGAAACTCATG-3'. The sequence for control
shRNA was as follows: 5'-AGGGGAGGGGAAAAATTT T-3'. Cells were selected with $1 \mu \mathrm{g} / \mathrm{ml}$ puromycin for 1 week. Following recovery for 7 days, subsequent assays were performed.

Western blotting. Proteins were extracted from HCC cells using radioimmunoprecipitation assay lysis buffer (Cell Signaling Technology, Inc., Danvers, MA, USA) containing protease and phosphatase inhibitors. Cell lysates were centrifuged $\left(12,000 \mathrm{x} \mathrm{g}\right.$ for $20 \mathrm{~min}$ at $\left.4^{\circ} \mathrm{C}\right)$ and the concentration was quantified using a Bradford assay. A total of $20 \mu \mathrm{g}$ proteins were separated by $10 \%$ SDS-PAGE and transferred to polyvinylidene difluoride membranes (EMD Millipore, Billerica, MA, USA). Membranes were blocked with 5\% milk at room temperature for $1 \mathrm{~h}$, and probed with primary and secondary antibodies. The immunoreactive protein bands were visualized using an Enhanced Chemiluminescence kit (Pierce; Thermo Fisher Scientific, Inc.). Antibodies to HMGCR (1:1,000; cat no. ab174830) and HSP90 (1:2,000; cat. no. ab13492) were purchased from Abcam (Cambridge, UK), and antibodies to myc tag (1:1,000; cat. no. sc-4084), GAPDH (1:5,000; cat. no. sc-293335), ubiquitin (1:750; cat. no. sc-8017) and Flag tag (1:1,000; cat. no. sc-51590) were purchased from Santa Cruz Biotechnology, Inc. (Dallas, TX, USA). Horseradish peroxidase-conjugated secondary antibodies, mouse IgG (1:1,000; cat. no. 7076) and rabbit $\operatorname{IgG}(1: 1,000$; cat. no. 7074) were obtained from Cell Signaling Technology, Inc.

Immunofluorescent staining. Cells were cultured on glass coverslips. Following $24 \mathrm{~h}$, cells were fixed with pre-chilled methanol at $-20^{\circ} \mathrm{C}$ for $5 \mathrm{~min}$. The following procedure was performed according to standard protocols. Briefly, after washing in PBS, the cells were blocked with PBS containing $3 \%$ bovine serum albumin (BSA; Shanghai Shenggong Biology Engineering Technology Service, Ltd., Shanghai, China) for $30 \mathrm{~min}$ at room temperature and then incubated with HMGCR (1:100; cat. no. ab174830) and HSP90 (1:200; cat. no. ab13492; both Abcam) antibodies in blocking buffer overnight at $4^{\circ} \mathrm{C}$, followed by incubation with a 1:1,000 dilution of the corresponding Alexa Fluor 488-conjugated secondary antibody (cat. no. 150077; Abcam) in blocking buffer at room temperature for $120 \mathrm{~min}$. Hoechst staining (1:2,000 dilution in PBS) was performed to label the nuclei. Fluorescence was monitored using an inverted confocal laser microscopy (Carl Zeiss AG, Oberkochen, Germany).

GST pull-down assay. The fusion protein GST-HSP90 was purified using Sepharose ${ }^{\circledR} 4 \mathrm{~B}$ beads (GE Healthcare Life Sciences, Little Chalfont, UK) according to the manufacturer's protocol. Cell lysates were prepared using cell lysis buffer (cat. no. 9803; Cell Signaling Technology, Inc.). Following centrifugation $\left(4^{\circ} \mathrm{C}, 10,000 \mathrm{x} \mathrm{g}, 20 \mathrm{~min}\right)$ the supernatant was incubated with $5 \mu \mathrm{g}$ GST fusion protein overnight at $4^{\circ} \mathrm{C}$. The Sepharose ${ }^{\circledR}$ 4B beads were added and incubated with cell lysate for an additional $4 \mathrm{~h}$. Subsequently, the beads were washed and the protein pulled down and detected by western blot analysis.

Statin treatment. Lovastatin was purchased from Sigma-Aldrich (Merck KGaA). Prior to treatment, Lovastatin was activated in $70 \%$ ethanol containing $0.1 \mathrm{~N} \mathrm{NaOH}$ at $50^{\circ} \mathrm{C}$ for $60 \mathrm{~min}$ and 
Table I. Association between expression of HSP90 and clinical features.

\begin{tabular}{|c|c|c|c|c|c|}
\hline \multirow[b]{2}{*}{ Characteristic } & \multirow[b]{2}{*}{ Total $(n=297)$} & \multicolumn{2}{|c|}{ HSP90 } & \multirow[b]{2}{*}{$\chi^{2}$} & \multirow[b]{2}{*}{ P-value } \\
\hline & & Low $(\mathrm{n}=61)$ & High $(n=236)$ & & \\
\hline Sex & & & & 0.412 & 0.521 \\
\hline Male & 266 & 56 & 210 & & \\
\hline Female & 31 & 5 & 26 & & \\
\hline Age (years) & & & & 0.597 & 0.44 \\
\hline$\geq 45$ & 182 & 40 & 142 & & \\
\hline$<45$ & 115 & 21 & 94 & & \\
\hline Tumor size & & & & 7.204 & $0.007^{\mathrm{b}}$ \\
\hline$\geq 6$ & 248 & 44 & 204 & & \\
\hline$<6$ & 49 & 17 & 32 & & \\
\hline Histological differentiation & & & & 2.879 & 0.09 \\
\hline Well & 258 & 49 & 209 & & \\
\hline Poor & 39 & 12 & 27 & & \\
\hline Organ metastasis & & & & 0.004 & 0.947 \\
\hline Yes & 74 & 15 & 59 & & \\
\hline No & 223 & 46 & 177 & & \\
\hline TNM & & & & 7.217 & $0.027^{\mathrm{a}}$ \\
\hline I & 40 & 13 & 27 & & \\
\hline II & 243 & 48 & 195 & & \\
\hline III, IV & 14 & 0 & 14 & & \\
\hline Tumor number & & & & 0.006 & 0.939 \\
\hline$\geq 2$ & 30 & 6 & 24 & & \\
\hline$\leq 1$ & 267 & 55 & 212 & & \\
\hline Lymph node metastasis & & & & 0.281 & 0.596 \\
\hline Yes & 19 & 3 & 16 & & \\
\hline No & 278 & 58 & 220 & & \\
\hline
\end{tabular}

Statistical analyses were performed using Pearson's $\chi^{2}$ test. ${ }^{\mathrm{P}} \mathrm{P}<0.05$ and ${ }^{\mathrm{b}} \mathrm{P}<0.001$. Score $<50$ was defined as 'Low' and score $\geq 50$ was defined as 'High'. HSP90, heat shock protein 90; TNM, tumor nodes metastasis.

then neutralized with $70 \%$ ethanol containing $0.1 \mathrm{~N} \mathrm{HCl}$ at room temperature for $1 \mathrm{~h}$. Activated lovastatin $(10 \mu \mathrm{M})$ was added to the medium the day after cells were plated, and the medium was changed every day. The cells were treated with lovastatin for a total of 7 days.

MTT assay. MTT assay was use to examine the growth of HCC cells. A total of 1,000 cells were seeded in 96-well plates containing $200 \mu \mathrm{l}$ DMEM and cultured for various durations. Every 2 days, $20 \mu \mathrm{l}$ MTT was added into the medium and incubated with cells for $4 \mathrm{~h}$. Then, the supernatant was cleared and cells were resolved with $200 \mu$ l DMSO. The absorabcne was measured at a wavelength of $540 \mathrm{~nm}$.

Anchorage-independent growth assay. For the soft agar assay, 6 -well plates were coated with a bottom layer $(0.5 \%$ agarose and $10 \%$ FBS in DMEM). Subsequently, the upper layer $(0.35 \%$ agarose and $10 \%$ FBS in DMEM) containing a total of 5,000 cells/well was plated. Following 14 days of incubation, the colonies were counted and measured using an inverted microscope (Carl Zeiss AG). All experiments were performed at least three times.

Immunohistochemistry. Tissue microarray was fixed with $4 \%$ formalin at $4^{\circ} \mathrm{C}$ overnight, embedded in paraffin, cut into $5 \mu \mathrm{m}$-thick consecutive sections and then deparaffinized at room temperature using xylene and ethanol. Antigen recovery was performed in sodium citrate solution ( $\mathrm{pH}$ 6.0) for $20 \mathrm{~min}$ at $95^{\circ} \mathrm{C}$. Subsequently, the sections were washed three times for $5 \mathrm{~min}$ in $0.01 \mathrm{~mol} / 1 \mathrm{PBS}$ containing $8 \mathrm{mmol} / 1 \mathrm{Na}_{2} \mathrm{HPO}_{4}$, $2 \mathrm{mmol} / 1 \mathrm{NaH}_{2} \mathrm{PO}_{4}$ and $150 \mathrm{mmol} / \mathrm{l} \mathrm{NaCl}$. The sections were then blocked for $1 \mathrm{~h}$ in $0.01 \mathrm{~mol} / \mathrm{l} \mathrm{PBS}$ containing $0.3 \%$ Triton X-100 and 5\% BSA (Shanghai Shenggong Biology Engineering Technology Service, Ltd.) at room temperature, followed by incubation with anti-HSP90 antibody (1:100; cat. no. ab13492; Abcam) at $4^{\circ} \mathrm{C}$ for at least $8 \mathrm{~h}$. Following brief washing with $0.01 \mathrm{~mol} / 1 \mathrm{PBS}$, sections were incubated with $0.01 \mathrm{~mol} / 1 \mathrm{PBS}$ containing horseradish peroxidase-conjugated anti-mouse IgG (1:500; cat. no. 7076; Cell Signaling Technology, Inc.) for $2 \mathrm{~h}$ at room temperature, followed by development with 
A

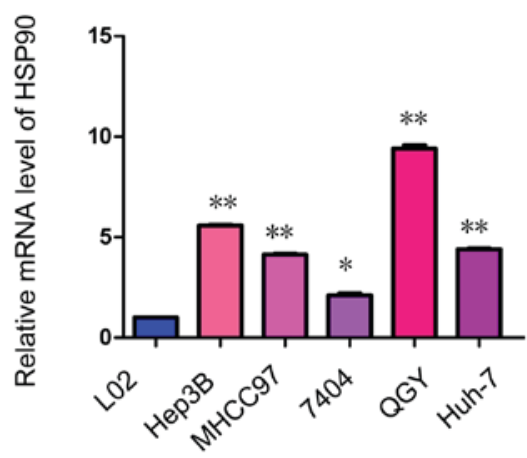

B
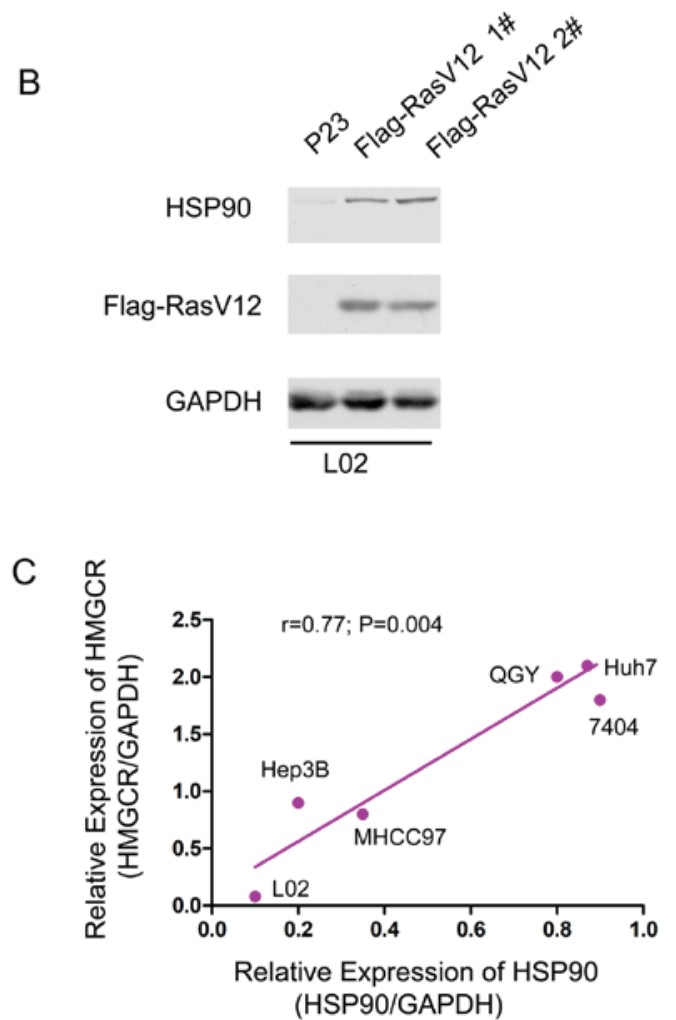

Figure 1. The expression levels of HSP90 are elevated in HCC cell lines. (A) The mRNA expression levels of HSP90 in HCC cell lines (Hep3B, MHCC97, 7404, QGY and Huh-7) and a normal cell line (L02) was examined using reverse transcription-quantitative polymerase chain reaction. $18 \mathrm{~S}$ was used as the reference gene. The mRNA expression levels of HSP90 in L02 was set as 1 . The experiment was performed three times. (B) Overexpression of RasV12 induced the expression of HSP90. (C) Positive association of HSP90 protein levels and HMGCR protein levels in HCC cell lines. The experiments were performed three times. HSP90, heat shock protein 90; HCC, hepatocellular carcinoma; HMGCR, 3-hydroxy-3-methylglutaryl-CoA reductase. ${ }^{*} \mathrm{P}<0.05,{ }^{* *} \mathrm{P}<0.01$ vs. $\mathrm{L} 02$.

$0.003 \% \mathrm{H}_{2} \mathrm{O}_{2}$ and $0.03 \%$ 3,3'-diaminobenzidine (DAB) in $0.05 \mathrm{~mol} / \mathrm{l}$ Tris-HCl ( $\mathrm{pH}$ 7.6). Immunohistochemistry for each sample was repeated three times. Slides were then developed with DAB and counterstained with hematoxylin at room temperature for $2 \mathrm{~min}$. The slides were evaluated using a light microscope.

Staining was assessed using the H-score method (21), according to the following equation: $\mathrm{H}$-score $=\Sigma(\mathrm{i} \times \mathrm{Pi})$. The staining intensity i) of the tumour cells was graded from 0-3, and $\mathrm{Pi}$ was the percentage of stained cells at each staining intensity. Tissues with a final HSP90 H-score <45 were classified as 'HSP90 low expression' and those with a final score $\geq 45$ were classified as 'HSP90 high expression'.

Immunoprecipitation assay. The immunoprecipitation assay was conducted as previously described (4). Cells were washed with ice-cold PBS and lysed in Tris-buffered saline $(\mathrm{pH} 7.4)$ containing $50 \mathrm{mM}$ Tris, $150 \mathrm{mM} \mathrm{NaCl}, 1 \% \mathrm{NP}-40,1 \mathrm{mM}$ EDTA, $1 \mathrm{mM} \mathrm{Na} \mathrm{VO}_{4}, 10 \mathrm{mM} \mathrm{NaF}, 2.5 \mathrm{mg} / \mathrm{ml}$ aprotinin and leupeptin, $1 \mathrm{mM} \beta$-glycerophosphate and 4-(2-aminoethyl) benzenesulfonyl fluoride hydrochloride and $10 \mathrm{mM}$ iodoacetate. Lysates were incubated on ice for $15 \mathrm{~min}$ prior to removal of cellular debris and nuclei via centrifugation at $10,000 \mathrm{x} \mathrm{g}$ for $20 \mathrm{~min}$ at $4^{\circ} \mathrm{C}$. Cell lysates were incubated overnight with the HMGCR antibody (1:1,000; cat. no. ab174830; Abcam) at $4^{\circ} \mathrm{C}$. IgG (1:1,000; cat. no. 7074; Cell Signaling Technology, Inc.) was used as control. Protein A Sepharose beads (GE Healthcare Life Sciences) in a 1:1 mixture with $50 \mathrm{mM}$ Tris buffer ( $\mathrm{pH} 7.0$ ) were added to the cell lysates and incubated for an additional $4 \mathrm{~h}$ at $4^{\circ} \mathrm{C}$. The immunoprecipitates were washed four times in Tris-buffered saline and boiled for $5 \mathrm{~min}$ in $40 \mu \mathrm{l}$ Laemmli buffer containing $0.02 \%$ blue bromophenol and $2 \% \beta$-mercaptoethanol.

Cell migration assay. Cell migration was evaluated using a Boyden chamber. The lower chamber was loaded with $0.152 \mathrm{ml}$ medium containing 10\% FBS. After $8 \mathrm{~h}$, cells that migrated to the lower surface of filters were detected with traditional hematoxylin and eosin staining for $5 \mathrm{~min}$ at room temperature. The migrated cells were counted under an inverted microscope and four fields of view were taken for each group. The experiments were repeated three times.

Overexpression of RasV12 in normal hepatic cells. The RasV12 plasmid (cat. no. 46746) was purchased from Addgene, Inc. and its coding sequence was inserted into a P23 lentiviral vector (cat. no. 100633; Addgene, Inc.) containing Flag tag and green fluorescent protein using BamHI and EcoRI. The lentivirus was produced using $293 \mathrm{~T}$ cells as previously described (6), and used to infect L02 at a multiplicity of infection of 1. Green fluorescent protein-positive cells were sorted using fluorescence-activated cell sorting and collected. Following a two-week recovery period, subsequent experiments were performed.

In vivo metastasis assay. Control cells (7404/P23) and HSP90 overexpressed cells (7404/Flag-HSP90) were labeled with the luciferase gene. A total of $1 \times 10^{6}$ cells were injected into nude mice through the tail vein. A total of 12 mice (male, five weeks old, $20 \mathrm{~g}$ ) were obtained from the Shanghai Laboratory Animal Center (Shanghai, China) and each group contained six mice. The mice were housed under standard conditions of $25^{\circ} \mathrm{C}$ with $27 \%$ humidity, free access to food and water, and a $12 \mathrm{~h}$ light/dark cycle. The mice were treated with lovastatin $(50 \mathrm{mg} / \mathrm{kg}$ ) every three days. Control mice were treated with ethanol. After 6 weeks, the mice were injected intraperitoneally with $150 \mathrm{mg} / \mathrm{kg}$ luciferin (Sigma-Aldrich; Merck KGaA) and after $5 \mathrm{~min}$ the metastasis foci were examined using Living Image 2.0 software (PerkinElmer, Inc., Waltham, MA, USA). The mice were sacrificed on the sixth week following imaging.

Statistics analysis. Each experiment was repeated at least three times. The data are presented as the means \pm standard 


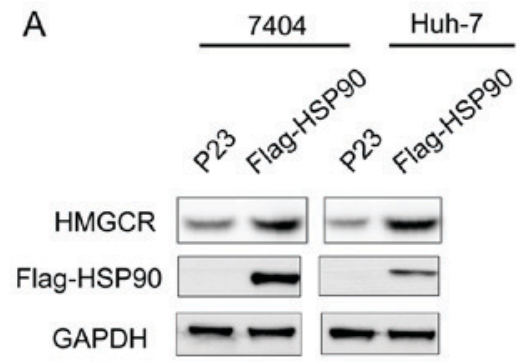

C

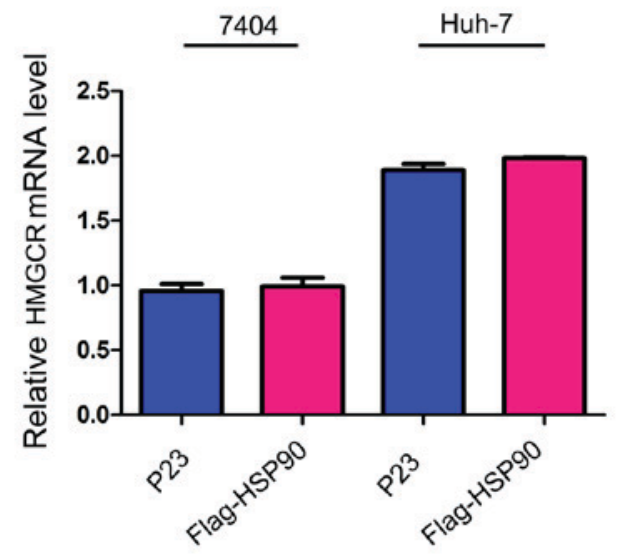

B
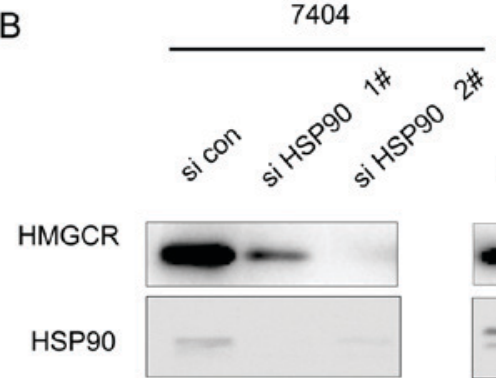

GAPDH

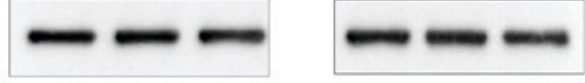

7404

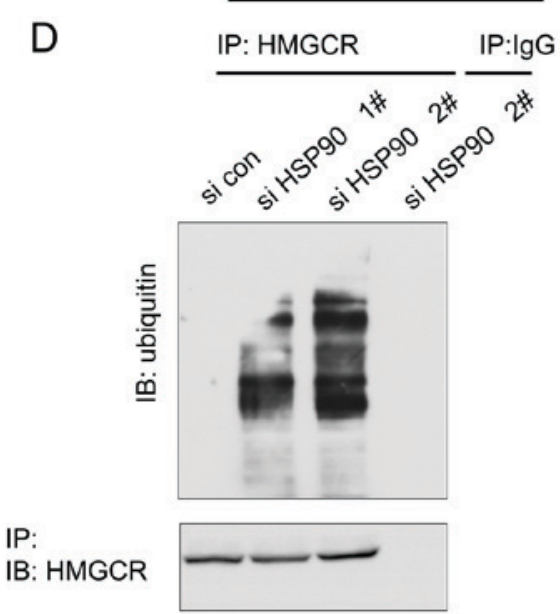

Figure 2. HSP90 upregulates the protein expression levels of HMGCR. (A) Overexpression of HSP90 increased the HMGCR protein expression levels in 7404 and Huh-7 cells. (B) Knock-down of HSP90 decreased the HMGCR protein expression levels in 7404 and Huh-7 cells. (C) Upregulation of HSP90 did not affect the HMGCR mRNA expression levels. (D) Knock-down of HSP90 in 7404 cells promoted the ubiquitination of HMGCR. The experiments were performed three times. HMGCR, 3-hydroxy-3-methylglutaryl-CoA reductase; HSP90, heat shock protein 90; si, small interfering RNA; con, control; IP, immunoprecipitated; IB, immunoblot.

deviation. Statistical analysis was performed using SPSS 15.0 (SPSS, Inc., Chicago, IL, USA). Statistical comparisons between two groups were performed using Student's t-test. For comparison among multiple groups, a two-way analysis of variance (ANOVA) was performed. Scheffe analysis was performed following ANOVA. For survival analysis, Kaplan-Meier curves were constructed and the P-value was determined using logrank test. For the association between the HSP90 expression and clinical features, the $\chi^{2}$ test was performed $\mathrm{P}<0.05$ was considered to indicate a statistically significant difference. The correlation between HSP90 and HMGCR expression was calculated using the Pearson's correlation coefficient.

\section{Results}

P90 is upregulated in HCC and is associated with clinical features. To examine the expression pattern of HSP90, this study used a HCC tissue microarray that included 297 patients with HCC. Immunohistochemistry staining was performed to evaluate the expression of HSP90 in HCC tissues. High HSP90 expression (score $>50$ ) was found in $~ 80 \%$ (236/297) clinical samples, and the expression of HSP90 was associated with tumor size and tumor nodes metastasis (TNM) stage (Table I).
In addition, the expression of HSP90 in a normal liver cell line (L02) and HCC cell lines (Hep3B, MHCC97, 7404, QGY and Huh-7) was investigated. Lower expression levels of HSP90 mRNA was observed in normal liver cells (L02), and higher HSP90 mRNA expression levels were observed in HCC cell lines (Fig. 1A). In addition, it was found that oncogenic RasV12 expression was associated with enhanced expression of HSP90, which may explain the upregulation of HSP90 in HCC tissues (Fig. 1B). Furthermore, the expression of HSP90 was positively associated with the protein expression level of HMGCR (Fig. 1C), suggesting that HSP90 may modulate the expression of HMGCR.

HSP90 elevates the protein expression level of HMGCR. Subsequently, the present study examined the modulation of HMGCR by HSP90. Overexpression of HSP90 in 7404 (P53 mutant) and Huh-7 (P53 mutant) cells significantly upregulated the expression of HMGCR (Fig. 2A). However, knock-down of HSP90 expression by two independent small interfering RNA sequences significantly decreased the protein level of HMGCR (Fig. 2B). In addition, the expression of HSP90 did not affect the mRNA expression levels of HMGCR (Fig. 2C), suggesting that HSP90 regulates the protein levels of HMGCR at the post-translation level. Consistent with this hypothesis, it 

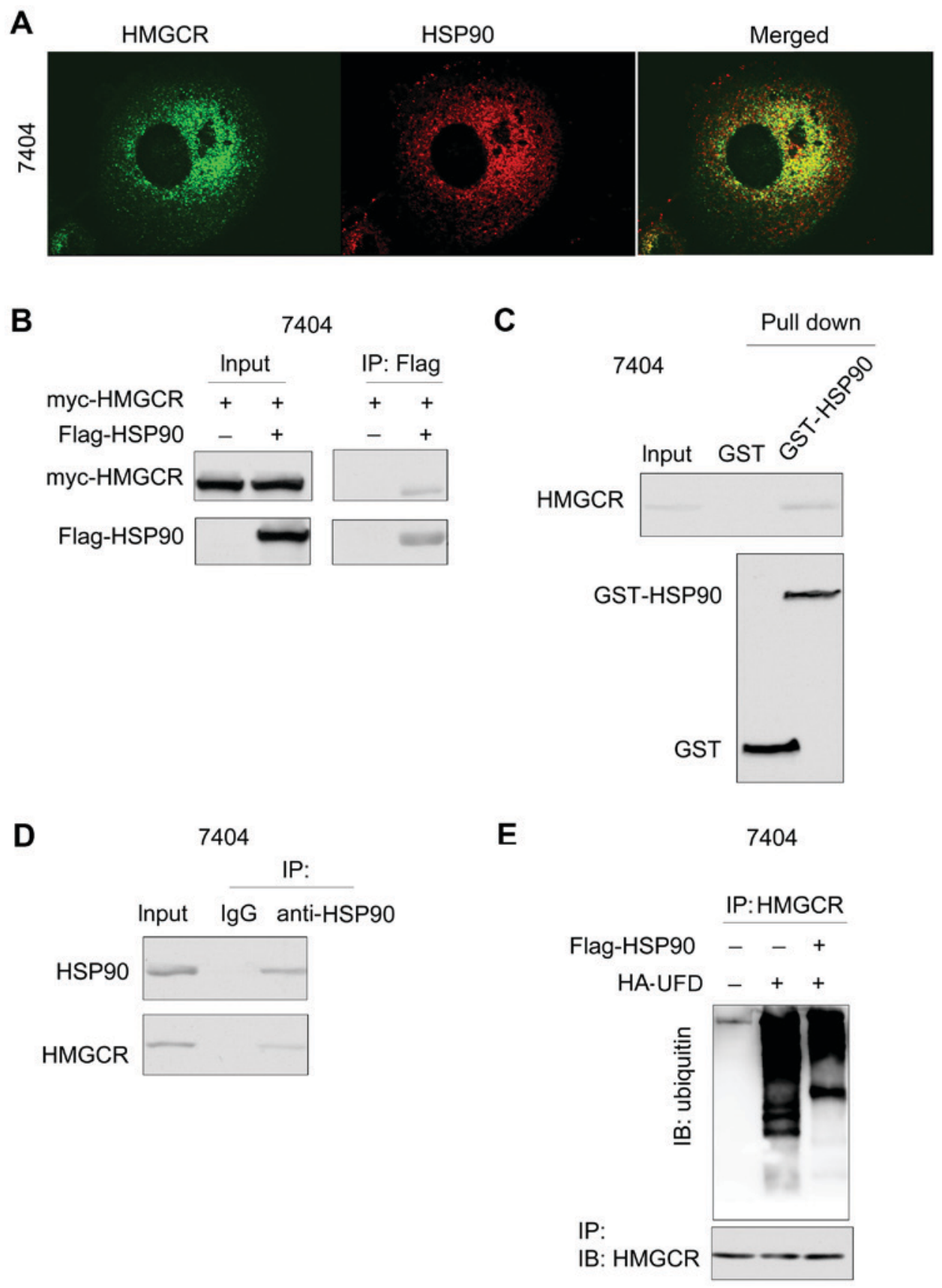

Figure 3. HSP90 interacts with HMGCR in 7404 cells. (A) Immunofluorescent staining for the co-localization of HSP90 and HMGCR in 7404 cells. (B) Exogenous expression of HSP90 (Flag-HSP90) and HMGCR (myc-HMGCR) formed a complex in 7404 cells, as determined by an immunoprecipitation assay. (C) GST-pull down assay was performed to demonstrate that the fusion protein GST-HSP90 and the endogenous HMGCR formed a complex in 7404 cells. (D) Endogenous HSP90 and HMGCR formed a complex. (E) Overexpression of HSP90 inhibited the ubiquitination of HMGCR induced by UFD. The experiments were performed three times. HMGCR, 3-hydroxy-3-methylglutaryl-CoA reductase; HSP90, heat shock protein 90; GST, glutathione S-transferase; UFD, ubiquitin fusion degradation.

was found that knock-down HSP90 promoted the ubiquitination of HMGCR (Fig. 2D), suggesting that HSP90 upregulates the protein levels of HMGCR by inhibiting its degradation.

HSP90 interacts with HMGCR. To explore the underlying mechanism by which HSP90 may inhibit the degradation of HMGCR, the interaction between HSP90 and HMGCR was examined. Immunofluorescent staining demonstrated the co-localization of HSP90 and HMGCR (Fig. 3A). Consistent with these observations, exogenously expressed HSP90 (Flag-HSP90) and HMGCR (myc-HMGCR) formed a complex in 7404 cells
(Fig. 3B). In addition, the fusion protein GST-HSP90 formed a complex with endogenously expressed HMGCR (Fig. 3C). Furthermore, endogenously expressed HMGCR interacted with HSP90 in the immunoprecipitation assay using anti-HSP90 antibody (Fig. 3D). These data demonstrated an interaction between HMGCR and HSP90. The study subsequently investigated the biological significance of the interaction between HSP90 and HMGCR. Overexpression of HSP90 attenuated the degradation of HMGCR induced by the expression of UFD (Fig. 3E). Taken together, these results suggested that HSP90 interacted with HMGCR and inhibited its degradation. 
A

7404

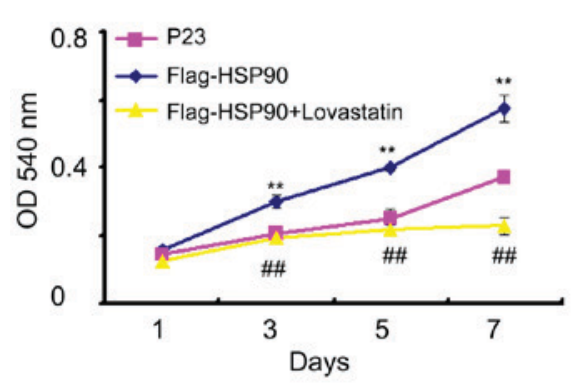

B
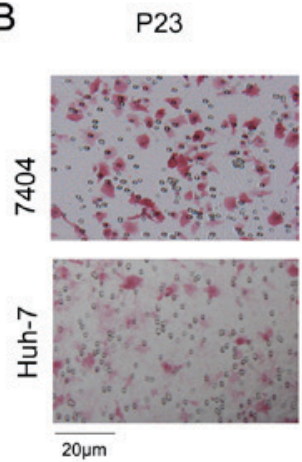

Flag-HSP90
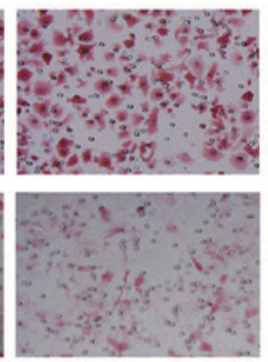

C
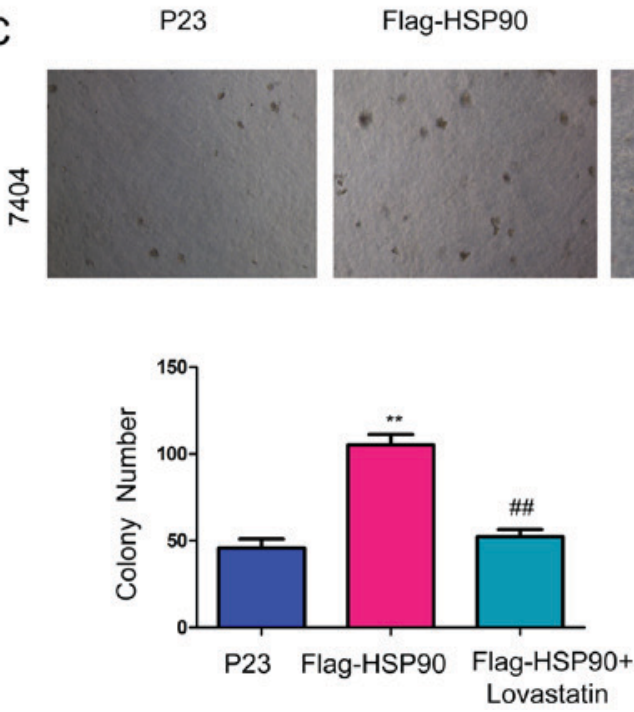

Huh-7

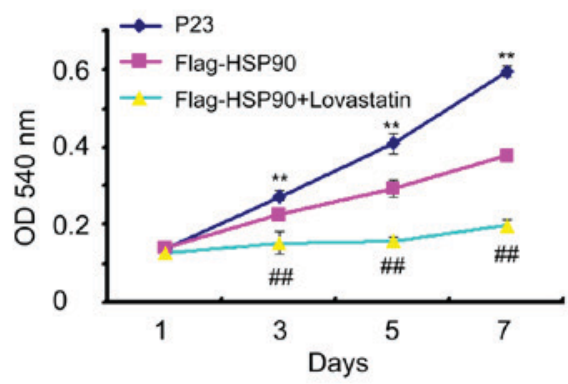

Flag-HSP90+

Lovastatin
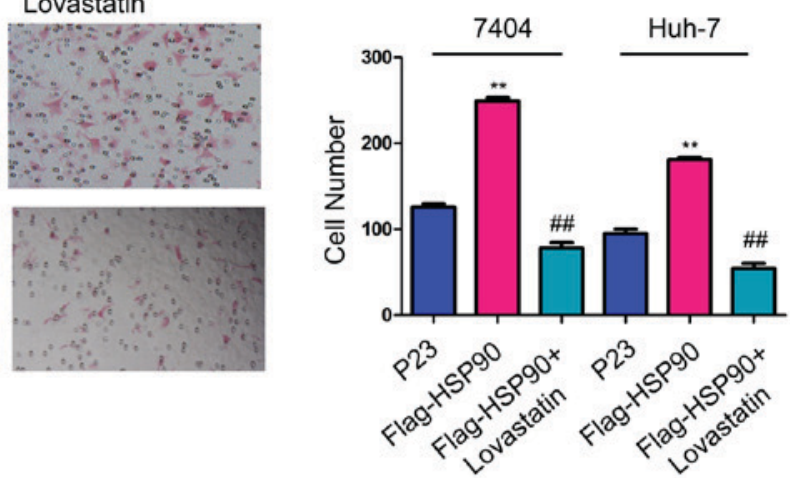

Flag-HSP90+ Lovastatin

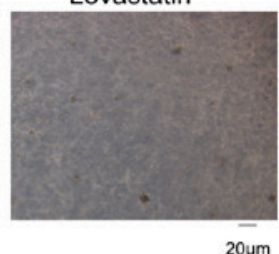

$20 \mu \mathrm{m}$

Figure 4. Lovastatin, an inhibitor of 3-hydroxy-3-methylglutaryl-CoA reductase, rescues the function of HSP90. (A) Lovastatin inhibited cell growth promoted by overexpression of HSP90. (B) Lovastatin inhibited cell migration promoted by overexpression of HSP90. Four fields of view were counted under the microscope for each group. (C) Lovastatin inhibited anchorage-independent growth of 7404 cells promoted by overexpression of HSP90. ${ }^{* *}$ P $<0.01$ vs. P23 group and ${ }^{\# \#} \mathrm{P}<0.01$ vs. Flag-HSP90 group. HSP90, heat shock protein 90; HMGCR, 3-hydroxy-3-methylglutaryl-CoA reductase.

Lovastatin, the inhibitor of HMGCR, inhibits the growth and migration of HCC cells. To study whether HMGCR mediated the biological functions of HSP90 in HCC cells, $10 \mu \mathrm{M}$ lovastatin, an inhibitor of HMGCR, was used to rescue the functions of HSP90. HSP90 promoted the growth (Fig. 4A), migration (Fig. 4B) and anchorage-independent growth of HCC cells (Fig. 4C), which was attenuated by the treatment of lovastatin. These results demonstrated that HSP90 regulated cell growth and migration by elevating the activity of HMGCR.

Lovastatin inhibits the metastasis of HCC cells in vivo. As presented in Table I, the expression of HSP90 was positively associated with the tumor TNM stage. Subsequently, this study examined whether HSP90 promoted the metastasis of HCC cells and whether its promotion on metastasis of HCC cells was dependent on the activity of HMGCR. As demonstrated in Fig. 5A, forced expression of HSP90 promoted the metastasis of 7404 cells, which were was injected into nude mice through the tail vein. Furthermore, the administration of lovastatin effectively blocked the metastasis of 7404 cells in vivo, which was demonstrated by the fluorescence intensity, photon counts and prolonged the survival of the mice (Fig. 5A-C). Taken together, these data suggested that HSP90 promoted the metastasis of HCC cells, which was dependent on the activity of HMGCR. 
A

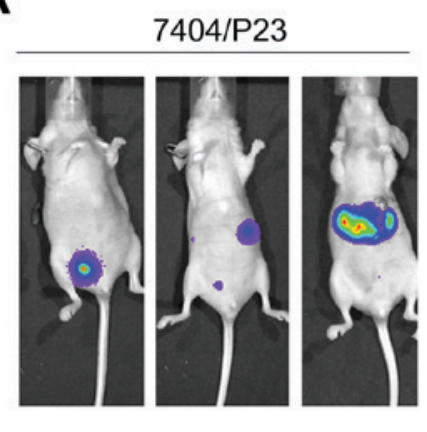

B

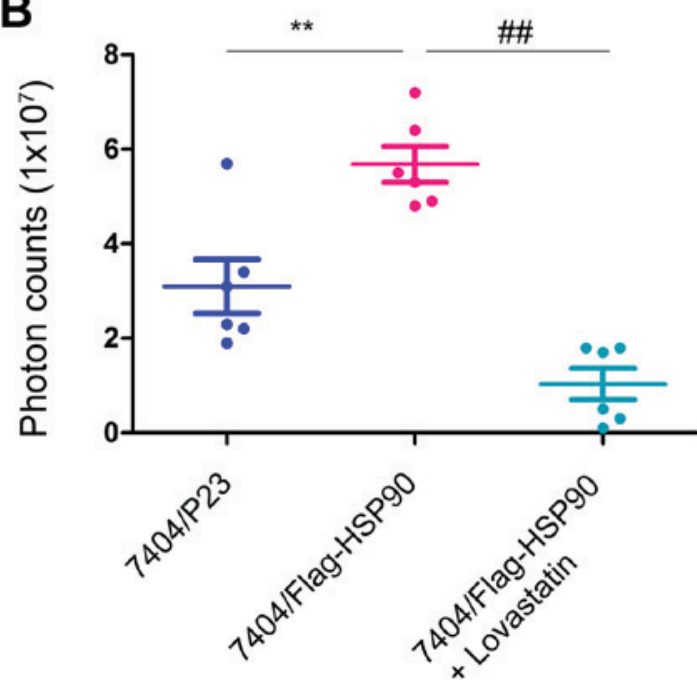

7404/Flag-HSP90

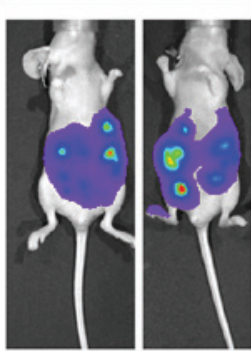

7404/Flag-HSP90

+ Lovastatin

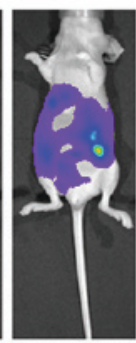

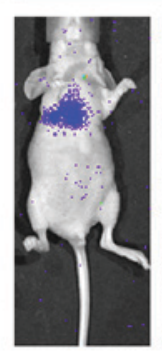

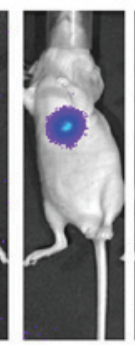

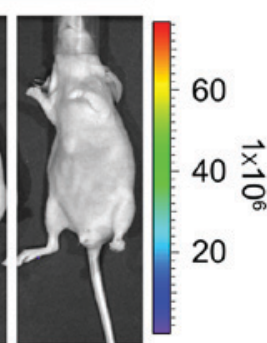

C $\quad+7404 /$ P 23

$+7404 /$ Flag-HSP90

- 7404/Flag-HSP90 + Lovastatin

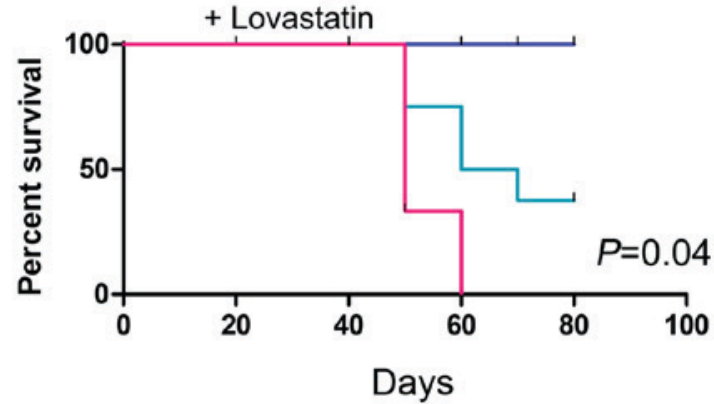

Figure 5. Lovastatin inhibits the metastasis of 7404 cells. (A) Lovastatin inhibited the metastasis of 7404 cells promoted by the overexpression of HSP90. The control cells (7404/P23) and 7404/Flag-HSP90 cells were injected into the tail vein of nude mice. After 42 days, metastasis of 7404 cells was examined using an in vivo imaging system. (B) Quantification (photon counts) in each group. ${ }^{* *} \mathrm{P}<0.01$ and ${ }^{\# \#} \mathrm{P}<0.01$. (C) Survival in each group. HSP90, heat shock protein 90 .

\section{Discussion}

Although several studies have demonstrated the oncogenic roles of HSP90 in the progression of HCC by promoting the proliferation and inhibiting apoptosis of cancer cells, the detailed underlying mechanism of the oncogenic roles of HSP90 in HCC remains poorly understood $(22,23)$. The findings of the current study demonstrated that the expression of HSP90 was significantly associated with clinical features. In addition, the expression of HSP90 was induced upon the activation of Ras signaling. Furthermore, HSP90 inhibited the degradation of HMGCR, the rate-limiting enzyme of the mevalonate pathway, which suggested a role for HSP90 in cholesterol metabolism. Notably, lovastatin, an inhibitor of HMGCR, reduced the malignant behavior of cancer cells via overexpression of HSP90 both in vitro and in vivo, suggesting that the clinical use of statins may be beneficial for HCC treatment. These data suggested that HSP90 promoted the progression of HCC by activating the mevalonate pathway.

An important finding of this study was the regulation of HMGCR degradation by HSP90. A previous study demonstrated that UFD and the E3 ligase GP78 cooperated to promote the ubiquitination and degradation of HMGCR (10). The present study demonstrated an interaction between HSP90 and HMGCR, and HSP90 inhibited the ubiquitination of HMGCR induced by UFD. Based on these observations, it is speculated that HSP90 may compete with UFD for binding with HMGCR.

Another notable finding was the induction of HSP90 by RasV12. Aberrant activation of RasV12 signaling is observed in $\sim 100 \%$ of HCC clinical samples (24), which may provide an explanation for the upregulation of HSP90 in HCC samples. In addition, HSP90 has been reported to positively regulate $\beta$-catenin/T-cell factor signaling (25), suggesting that HSP90 may be an important protein for various signaling pathways.

In the current study, lovastatin, an inhibitor of HMGCR, exhibited anti-cancer activity in HCC cells. Lovastatin impaired the growth, migration and metastasis of HCC cells driven by HSP90. Combination of 17-dimethylaminoethylamino-17-demethoxygeldanamycin (an inhibitor of HSP90) and lovastatin (the inhibitor for HMGCR) may be useful for HCC therapy.

In conclusion, the present study may have elucidated a mechanism through which HSP90 promotes the progression of HCC, and HSP90 may be a promising therapeutic target. Further investigation of the therapeutic effects of HSP90 and HMGCR inhibitors for HCC will provide novel insights.

\section{Acknowledgements}

Not applicable. 


\section{Funding}

This study was supported by the Nature Science Foundation of Inner Mongolia (grant no. 2015MS08144).

\section{Availability of data and materials}

All data generated or analyzed during this study are included in this published article.

\section{Authors' contributions}

LD and RG designed the present study. LX, CZ, HL and ZC performed the experiments.

\section{Ethics approval and consent to participate}

This study was approved by the ethics committee of Inner Mongolia People's Hospital, and written informed consent was obtained from patients.

\section{Patient consent for publication}

Written informed consent was obtained from patients.

\section{Competing interests}

The authors declare that they have no competing interests.

\section{References}

1. Siegel RL, Miller KD and Jemal A: Cancer statistics, 2016. CA Cancer J Clin 66: 7-30, 2016.

2. Dai XY, Zhuang LH, Wang DD, Zhou TY, Chang LL, Gai RH, Zhu DF, Yang B, Zhu H and He QJ: Nuclear translocation and activation of YAP by hypoxia contributes to the chemoresistance of SN38 in hepatocellular carcinoma cells. Oncotarget 7: 6933-6947, 2016.

3. Hanahan D and Weinberg RA: Hallmarks of cancer: The next generation. Cell 144: 646-674, 2011.

4. Hashemi M, Hoshyar R, Ande SR, Chen QM, Solomon C, Zuse A and Naderi M: Mevalonate cascade and its regulation in cholesterol metabolism in different tissue in health and disease. Curr Mol Pharmacol 10: 13-26, 2016.

5. Gabor KA and Fessler MB: Roles of the mevalonate pathway and cholesterol trafficking in pulmonary host defense. Curr Mol Pharmacol 10: 27-45, 2016.

6. Hashimoto A, Oikawa T, Hashimoto S, Sugino H, Yoshikawa A, Otsuka Y, Handa H, Onodera Y, Nam JM, Oneyama C et al: P53and mevalonate pathway-driven malignancies require Arf6 for metastasis and drug resistance. J Cell Biol 213: 81-95, 2016.

7. Zahra Bathaie S, Ashrafi M, Azizian M and Tamanoi F: Mevalonate Pathway and Human Cancers. Curr Mol Pharmacol 10: 77-85, 2017.
8. Chushi L, Wei W, Kangkang X, Yongzeng F, Ning X and Xiaolei C: HMGCR is up-regulated in gastric cancer and promotes the growth and migration of the cancer cells. Gene 587: 42-47, 2016.

9. Mukherjee M, Basu Ball W and Das PK: Leishmania donovani activates SREBP2 to modulate macrophage membrane cholesterol and mitochondrial oxidants for establishment of infection. Int J Biochem Cell Biol 55: 196-208, 2014.

10. Cao J, Wang J, Qi W, Miao HH, Wang J, Ge L, DeBose-Boyd RA, Tang JJ, Li BL and Song BL: Ufd1 is a cofactor of gp78 and plays a key role in cholesterol metabolism by regulating the stability of HMG-CoA reductase. Cell Metab 6: 115-128, 2007.

11. Singh R, Yadav V, Kumar S and Saini N: MicroRNA-195 inhibits proliferation, invasion and metastasis in breast cancer cells by targeting FASN, HMGCR, ACACA and CYP27B1. Sci Rep 5: 17454, 2015.

12. Richter K and Buchner J: Hsp90: Twist and fold. Cell 127: 251-253, 2006.

13. Sawai A, Chandarlapaty S, Greulich H, Gonen M, Ye Q, Arteaga CL, Sellers W, Rosen N and Solit DB: Inhibition of Hsp90 down-regulates mutant epidermal growth factor receptor (EGFR) expression and sensitizes EGFR mutant tumors to paclitaxel. Cancer Res 68: 589-596, 2008.

14. Citri A, Gan J, Mosesson Y, Vereb G, Szollosi J and Yarden Y: Hsp90 restrains ErbB-2/HER 2 signalling by limiting heterodimer formation. EMBO Rep 5: 1165-1170, 2004.

15. Sato S, Fujita N and Tsuruo T: Modulation of Akt kinase activity by binding to Hsp90. Proc Natl Acad Sci USA 97: 10832-10837, 2000.

16. Minet E, Mottet D, Michel G, Roland I, Raes M, Remacle J and Michiels C: Hypoxia-induced activation of HIF-1: Role of HIF-1alpha-Hsp90 interaction. FEBS Lett 460: 251-256, 1999.

17. Lee YJ, Curetty L, Hou ZZ, Kim SH, Kim JH and Corry PM: Effect of $\mathrm{pH}$ on quercetin-induced suppression of heat shock gene expression and thermotolerance development in HT-29 cells. Biochem Biophys Res Commun 186: 1121-1128, 1992.

18. Zhou CC, Yang F, Yuan SX, Ma JZ, Liu F, Yuan JH, Bi FR, Lin KY, Yin JH, Cao GW, et al: Systemic genome screening identifies the outcome associated focal loss of long noncoding RNA PRAL in hepatocellular carcinoma. Hepatology 63: 850-863, 2016.

19. Zhao S, Li H, Jiang $\mathrm{C}$, Ma T, Wu C, Huo Q and Liu H: 17-Demethoxy-reblastatin, an Hsp90 inhibitor, induces mitochondria-mediated apoptosis through downregulation of Mcl-1 in human hepatocellular carcinoma cells. J Bioenerg Biomembr 47: 373-381, 2015.

20. Livak KJ and Schmittgen TD: Analysis of relative gene expression data using real-time quantitative PCR and the 2(-Delta Delta C(T)). Methods 25: 402-408, 25, 2001.

21. Cai Z, Qian ZY, Jiang H, Ma N, Li Z, Liu LY, Ren XX, Shang YR, Wang JJ, Li JJ, et al: HPCL3s promotes metastasis of hepatocellular carcinoma by activating $\beta$-catenin/TCF signaling. Cancer Res 78: 2536-2549, 2018

22. Wang C, Zhang Y, Guo K, Wang N, Jin H, Liu Y and Qin W: Heat shock proteins in hepatocellular carcinoma: Molecular mechanism and therapeutic potential. Int J Cancer 138: 1824-1834, 2016.

23. Liu X, Chen S, Tu J, Cai W and Xu Q: HSP90 inhibits apoptosis and promotes growth by regulating HIF-1alpha abundance in hepatocellular carcinoma. Int J Mol Med 37: 825-835, 2016.

24. Neuzillet C, Tijeras-Raballand A, de Mestier L, Cros J, Faivre S and Raymond E: MEK in cancer and cancer therapy. Pharmacol Ther 141: 160-171, 2014

25. CooperLC,PrinslooE,Edkins ALandBlatch GL:Hsp90alpha/beta associateswith theGSK3beta/axin1/phospho-beta-catenincomplex in the human MCF-7 epithelial breast cancer model. Biochem Biophys Res Commun 413: 550-554, 2011. 